\title{
Algorithm for managing severe ulcerative colitis
}

\author{
Vineet Ahuja ${ }^{1}$, Ajay Kumar' ${ }^{2}$, Rakesh Kochhar ${ }^{3}$
}

ABSTRACT

Dept of Gastroenterology, ${ }^{1}$ All India Institute of Medical Sciences, New Delhi

${ }^{2}$ Apollo Indraprastha Hospitals, New Delhi,

${ }^{3}$ Post Graduate Institute of Medical Education and Research, Chandigarh India

Corresponding author:

Vineet Ahuja

Email: vinsahuja@hotmail.com
Acute severe ulcerative colitis as defined by Truelove Witt's criteria is a medical emergency which requires hospitalization. Patient requires close clinical monitoring with assessment of daily vital parameters, stool frequency, serum electrolytes and daily abdominal examination. Intravenous corticosteroids are the mainstay of therapy. Approximately two thirds of patients will respond to steroids. Response to steroids should be assessed at day 3 of admission and partial/ non-responders should be considered for alternative medical therapy/ surgery. Medical rescue therapies include intravenous cyclosporin and infliximab. This article provides an time bound step up algorithm for management of acute severe ulcerative colitis.

Keywords: Acute severe colitis, Ulcerative colitis, management, algorithm
Acute severe ulcerative colitis is a medical emergency and carries a mortality rate of $1-2.9 \%$. Effective treatment relies on two management principles:

a) Multidisciplinary management including a gastroenterologist as well as a colorectal surgeon

b) Time Framed Step Up strategy as prolonged steroid therapy or delay in surgical therapy can be life threatening.

This article presents a time bound framework, which looks at stepwise management of acute severe ulcerative colitis. Literature details have been spared as they have already been described in reviews within this supplement.

\section{Step 1: Categorizing a patient as having Acute Severe Ulcerative Colitis (ASUC)}

In a diagnosed case of ulcerative colitis, Truelove and Witts' classification still remains most appropriate to diagnose acute severe ulcerative colitis

\begin{tabular}{ll}
\hline Parameter & Severe Colitis \\
\hline Number of bloody stools per day $(\mathrm{n})$ & $>6$ \\
and at least one of the following: & $>37.8$ \\
Temperature $\left({ }^{\circ} \mathrm{C}\right)$ & $>90$ \\
Heart rate (beats per minute) & $<10.5$ \\
Haemoglobin $(\mathrm{g} / \mathrm{dl})$ & $>30$ \\
Erythrocyte sedimentation rate $(\mathrm{mm} / \mathrm{h})$ & \\
\hline
\end{tabular}

(C) Tropical Gastroenterology 2014
Step 2: Does every patient of acute severe ulcerative colitis require hospitalization?

i) All patients with ASUC requiring intravenous steroids should be hospitalized.

ii) The term " fulminant colitis " is no longer used routinely. Fulminant colitis denotes a very severe form of disease as evidenced by 10 or more bloody stools with systemic features of activity and colonic dilatation on plain abdominal Xray. A patient with fulminant colitis should be immediately hospitalized.

iii) ASUC patients presenting with toxic megacolon or features of peritonism should be immediately hospitalized.

iv) However if a patient has not been on oral steroids and has progressed from a mild or moderate to severe disease then a trial of oral steroids may be given without immediate hospitalization .

\section{Step 3: Day 1 Management}

Investigations:

i) Haemogram including ESR, CRP, serum electrolytes, blood sugar, serum albumin, liver function tests and renal function tests

ii) Viral markers: HbsAg, IgG anti HBc, anti HCV and HIV 
iii) Stool tests:

a) Stool assay for Clostridium difficile cytotoxin (both A and B)

b) Stool culture for bacterial pathogens

c) Stool examination for parasitic infestations

iv) Baseline abdominal radiographs

v) Flexible unprepared sigmoidoscopy with minimal air insufflation to confirm disease, assess endoscopic severity and rectal biopsies for CMV colitis. CMV testing may be done at tertiary care centers only as facilities may not be available at all centers.

vi) Investigations best avoided:

a) Barium enema

b) Full length colonoscopy

c) Colon biopsy from a site more than $10 \mathrm{cms}$ from anal verge

\section{Therapy}

Patients with ASUC should be treated in hospital jointly by a gastroenterologist and a colorectal surgeon.

\section{Definitive Therapy}

Steroid therapy:

i) First-line medical therapy for patients should be intravenous corticosteroids hydrocortisone $100 \mathrm{mg}$ four times or methylprednisolone $60 \mathrm{mg}$ daily.

ii) Divided dose injection is as effective as continuous infusion

iii) Treatment is usually given for about 5 days, since extending therapy beyond 7 to 10 days carries no benefit.

iv) In patients with severe osteoporosis, uncontrolled diabetes mellitus or history of steroid psychosis or intolerant of intravenous steroids, monotherapy with intravenous cyclosporin (to achieve a minimum therapeutic concentration) is an option.

\section{Antibiotics:}

i) Routine use of antibiotics is not recommended.

ii) Antibiotics are given in following situations :i) if infection is considered (such as in an acute first attack of short duration, or after recent admission to hospital or presence of leucocytosis), ii) severe toxaemia iii) features of toxic megacolon are present. iii) Intravenous quinolone and metronidazole combination is the preferred choice.

\section{Adjunct Therapy:}

i) Intravenous fluid and electrolyte replacement to correct and prevent dehydration or electrolyte imbalance.

ii) Prophylaxis for thromboembolic complications

iii) Blood transfusion to maintain a haemoglobin of $10 \mathrm{~g} / \mathrm{dl}$

Role of 5-aminosalicylates:

iv) Topical therapy in form of $5 \mathrm{ASA}$ /steroids enemas or suppositories is advisable, although no trials are available which have evaluated the role of topical therapy in ASUC.

v) Physician experience suggests that oral 5ASA may be discontinued temporarily as it may not provide any additional anti- inflammatory benefit over intravenous steroids.

\section{Nutrition:}

i) Patients should be offered a normal diet or enteral nutrition unless such a diet is not tolerated.

ii) TPN is not effective as primary therapy. TPN should be considered only in malnourished patients who cannot tolerate oral intake or enteral nutrition.

\section{Drugs which should not be given:}

i) Drugs which may slow down intestinal transit and precipitate colonic dilation should be avoided. These include: anticholinergics, antidiarrhoeal agents, NSAIDs and opiates.

\section{Monitoring:}

i) Bedside stool monitoring chart to be maintained .

ii) Surgical consultation should be obtained for all patients with systemic toxicity or megacolon.

\section{Patient Counselling:}

Often neglected but a key factor for effective management is making the patient and family aware of the possible course of disease and therapeutic options including surgery. An informed 
patient will help the treating physician in making choice for further management.

\section{Step 4}

\section{Day 2 Management:}

\section{Daily Monitoring:}

i) Physical examination daily to evaluate abdominal tenderness, presence of rebound tenderness and bowel sounds.

ii) Recording of vital signs four times daily and noted in monitoring chart.

iii) Stool chart (to record number, consistency i.e. liquid versus solid stool, presence or absence of blood and nocturnal frequency of bowel movements)

\section{Investigations:}

i) Haemogram, ESR, CRP, serum electrolytes, serum albumin, and glucose every 24 hours.

ii) If there are signs of colonic distension and/or there is significant deterioration in clinical condition or blood parameters then abdominal $\mathrm{X}$ ray should be done

\section{Day 3-5 Management:}

Joint medical and surgical management is appropriate.

\section{Investigations:}

Monitoring and investigations as planned for Day 2 are to be similarly carried out on day 3, 4 and 5 .

In addition, monitoring after 72 hours of intravenous steroid institution includes:

a) Decision Making Tool: Evaluating for Oxford (Travis) Index

Non Responders: A stool frequency $>4$ and CRP $>45 \mathrm{mg} / \mathrm{L}$ on day 3; or a stool frequency $>8 / 24$ hours.

Responders: A stool frequency of less than 3 at Day 3.

b) Chances of colectomy are much higher in non/incomplete responders

\section{Non or Incomplete Responders:}

In non responders or incomplete responders, following measures should be planned:

i) Patient Education and Counselling: Explaining the patient and family the options of surgery or medical rescue therapy with cyclosporin or infliximab and guide the patient to participate in an informed decision making process.

ii) If a patient is planned for cyclosporin then investigations to be done include: serum magnesium, serum cholesterol and serum creatinine

iii) If a patient is planned for biological therapy then the following investigations should be done: High resolution contrast tomography of chest to exclude active TB infection, Monteux test or Interferon gamma release assay should be done.

iv) If a patient is planned for biological therapy or surgery and serum albumin is low then in both instances intravenous albumin should be supplemented so as to build up albumin levels.

\section{Step 5: Day 5 and beyond Time Bound Decisions:}

i) A decision regarding surgical or medical rescue therapy (Infliximab or cyclosporin) should be made within 5-7 days after initiation of intravenous steroids.

ii) Response to infliximab or cyclosporin should also be made within 5-7 days after initiation

iii) Either intravenous cyclosporin or infliximab is an appropriate choice for selected patients who have failed intravenous corticosteroid therapy.

iv) Factors which may dictate choice of either agent include: For infliximab, major impediment is affordability; For cyclosporin, major impediments are lack of physician experience in its usage and if the patient is already on azathioprine. Cyclosporin may be more beneficial in azathioprine naïve ASUC patients.

iv) It is best for centers with inadequate experience to refer patients at this stage to centers which have appropriate experience and support in use of cyclosporin and infliximab.

\section{Cyclosporin use:}

i) Cyclosporin should not be used if cholesterol $<115 \mathrm{mg} / \mathrm{dL}$ or magnesium $<1.4 \mathrm{mEq} / \mathrm{L}$. It should also be avoided in presence of hypertension, renal impairment, epilepsy, sepsis, age $>80$ years. Magnesium, cholesterol, and creatinine should be measured at baseline and within 48 hours of starting cyclosporin.

ii) Cyclosporin should be administered in a dose of $2 \mathrm{mg} / \mathrm{kg} /$ 
day intravenously aiming for trough levels $150-250 \mathrm{ng} / \mathrm{mL}$.

iii) Blood pressure and renal function should be monitored and cyclosporin should be stopped if serum creatinine rises $>25 \%$.

iv) Cyclosporin should be stopped if there is no improvement in 7 days

v) In responders, intravenous cyclosporin should be switched to oral cyclosporine $4 \mathrm{mg} / \mathrm{kg} /$ day twice daily. Monitoring of trough levels (150-250 ng/mL) should be regularly done. Azathioprine, which should be started along with oral cyclosporin. Cyclosporin should be stopped after 3 months.

\section{Infliximab Therapy:}

i) Patients who respond to a single infusion of infliximab 5 $\mathrm{mg} / \mathrm{kg}$ IV should be given two additional induction doses at 2 and 6 weeks, followed by maintenance infliximab therapy.

ii) If patients are not on azathioprine, it can be started by day 5 after the initiation of infliximab injection in ASUC

ii) Sequential rescue therapy in patients with cyclosporin non responders with infliximaband vice versa should be avoided.

\section{Tacrolimus (FK506):}

Tacrolimus is a calcineurin inhibitor, which can be an alternative agent. Tacrolimus $(0.1 \mathrm{mg} / \mathrm{kg}$ body weight per day) may be administered orally and initially intravenously $(0.01 \mathrm{mg} / \mathrm{kg}$ body weight per day), aiming for serum trough levels of $4-8 \mathrm{ng} / \mathrm{mL}$.

\section{Surgery:}

i) Patients who fail to respond to infliximab or cyclosporin within 5-7 days have a poor outcome and surgery is advisable.

ii) When surgery is required, total or subtotal colectomy with end ileostomy is the procedure of choice.

iii) Pouchitis frequency is much less when done by an experienced colorectal surgeon who does at least 10 pouch surgeries every year.

The described algorithm lays down the flow of investigations and therapeutic strategies in a timed format (Figure $\mathbf{1}$ and 2).

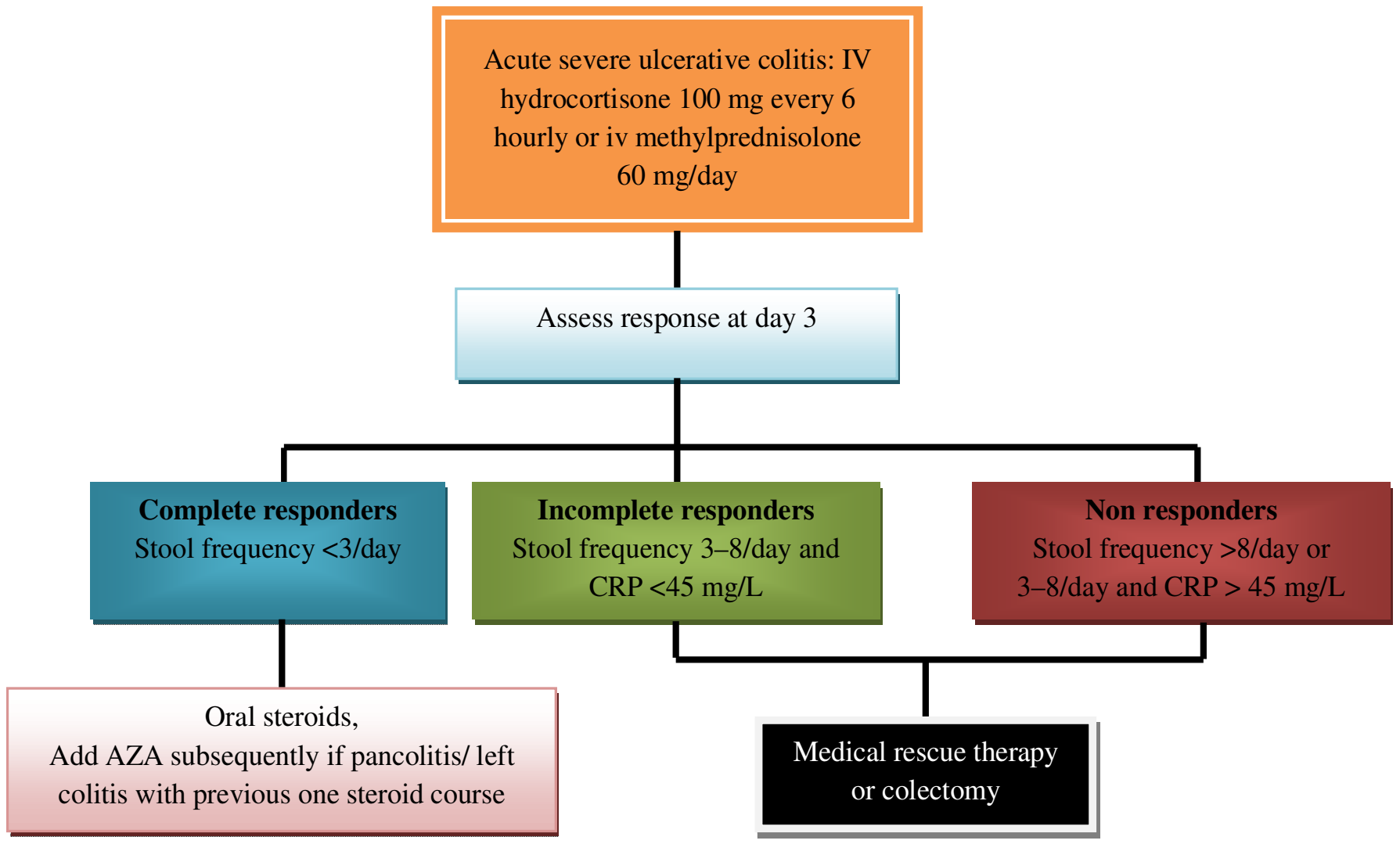

Figure 1: Algorithm for treatment decisions for patients with acute severe UC on intensive steroid therapy 


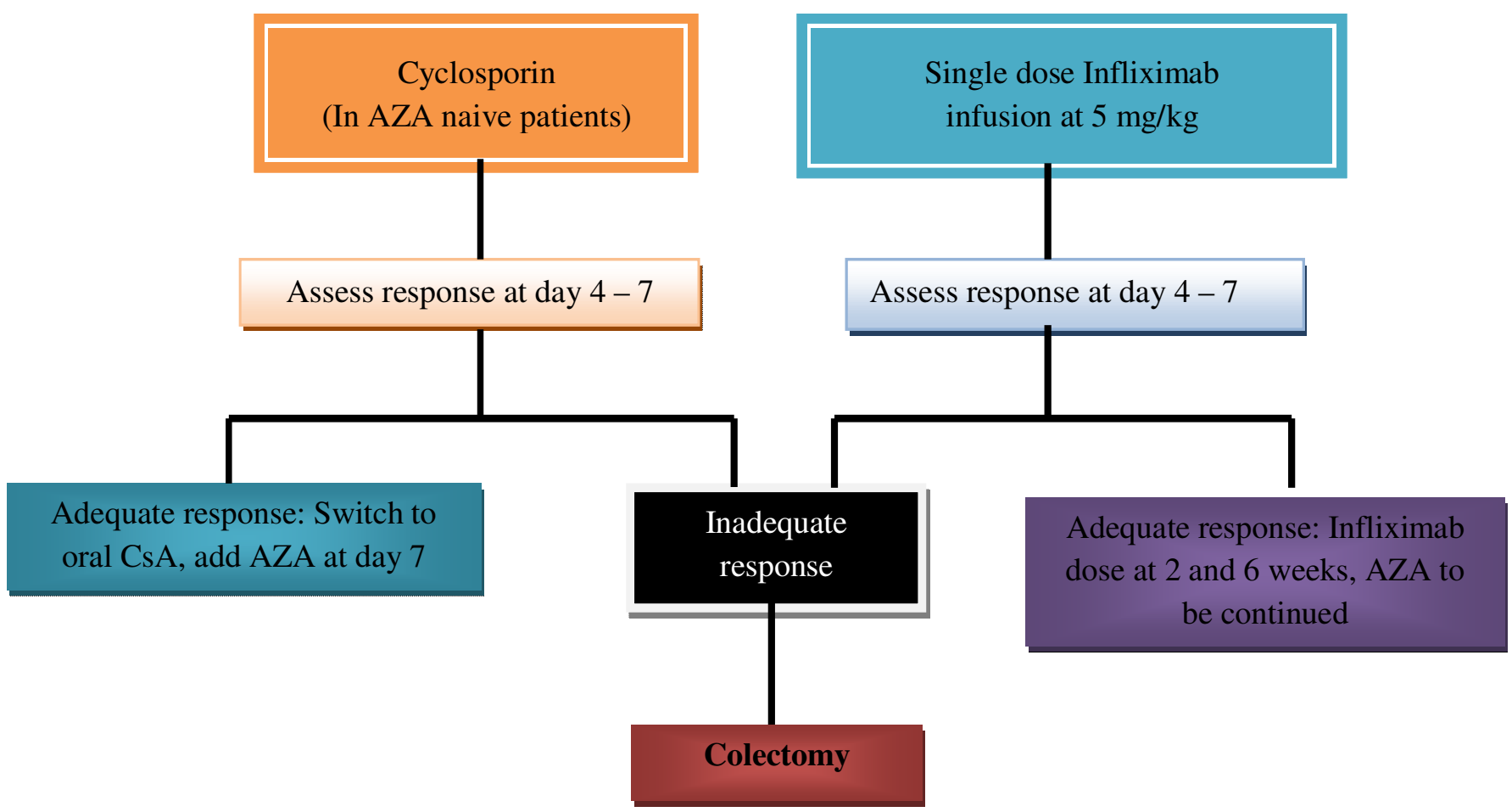

Figure 2: Algorithm for medical rescue therapy after failure of response to intravenous steroids

\section{Suggested reading:}

1. Dignass A, Van Assche G, Lindsay JO, Lemann M, Soderholm J, ColombelJF,et al. The second European evidence-based Consensus on the diagnosis and management of Crohn's disease: Current management. J Crohns Colitis. 2010;4:28-62.

2. Turner D, Travis SP, Griffiths AM, Ruemmele FM, Levine A, Benchimol EI, et al. European Crohn's and Colitis Organization; Porto IBD Working Group, European Society of Pediatric
Gastroenterology, Hepatology, and Nutrition. Consensus for managing acute severe ulcerative colitis in children: a systematic review and joint statement from ECCO, ESPGHAN, and the Porto IBD Working Group of ESPGHAN. Am J Gastroenterol. 2011;106:574-88.

3. Van Assche G, Vermeire S, Rutgeerts P. Management of acute severe ulcerative colitis. Gut. 2011;60:130-3. 